\title{
Assessment of the insecticidal activity of oral afoxolaner against Phlebotomus perniciosus in dogs
}

\author{
Nadège Perier ${ }^{1}$, Wilfried Lebon ${ }^{1}$, Leon Meyer ${ }^{2}$, Noua Lekouch $^{2}$, Nesrine Aouiche $^{1}$, and Frédéric Beugnet ${ }^{1, *}$ \\ ${ }^{1}$ Boehringer Ingelheim Animal Health, 29 avenue Tony Garnier, 69007 Lyon, France \\ ${ }^{2}$ Clinvet, Douar Dbabej Beni Yekhlef, 28815 Mohammedia, Morocco
}

Received 9 September 2019, Accepted 16 October 2019, Published online 5 November 2019

\begin{abstract}
Twelve healthy dogs were included in this laboratory efficacy study. Six dogs were randomly allocated based on body weight to an untreated control group and six to an afoxolaner (NexGard ${ }^{\circledR}$ ) treated group. In the treatment group, afoxolaner was administered orally on Day 0 in accordance with label instructions. On Days 1, 14 and 28 , each dog was exposed to 60 unfed female and 10 male Phlebotomus perniciosus sandflies for $1 \mathrm{~h}$. At the end of each exposure period, sandflies were counted and assessed for viability and feeding status. There was no statistical difference in mortality $(0.0-5.4 \%)$, nor in feeding proportion (61.6-78\%) between the control and the treated groups at all 1-h post-exposure assessments. After collection, live fed and unfed sandflies were kept for viability assessments at 48 and $72 \mathrm{~h}$ post-exposure. In the untreated control group, the average percentages of live, fed, female sandflies after exposure, on Days 1, 14 and 28, ranged from $51 \%$ to $74 \%$ at $48 \mathrm{~h}$ and from $46 \%$ to $57 \%$ at $72 \mathrm{~h}$, demonstrating model robustness over the 28 days of the study. Significantly fewer live fed sandflies were recorded for the afoxolaner treated group $(p<0.01)$. The insecticidal efficacy was $100 \%, 95.9 \%$ and $75.2 \%$ at $48 \mathrm{~h}$ post Days 1,14 and 28 exposures, respectively, and $100 \%, 100 \%$ and $86.3 \%$ at $72 \mathrm{~h}$ post Days 1,14 , and 28 exposures, respectively. A single administration of oral afoxolaner $\left(\mathrm{NexGard}^{\circledR}\right)$ to dogs significantly killed P. perniciosus sandflies 48 and $72 \mathrm{~h}$ after blood feeding for 1 month.
\end{abstract}

Key words: Phlebotomus perniciosus, Sandfly, Insecticide, Afoxolaner, NexGard ${ }^{\circledR}$, Dog.

Résumé - Évaluation de l'activité insecticide de l'afoxolaner par voie orale contre Phlebotomus perniciosus chez le chien. Douze chiens en bonne santé ont été inclus dans cette étude d'efficacité en laboratoire. Six chiens ont été répartis au hasard en fonction de leur poids corporel dans un groupe témoin non traité et six dans un groupe traité par afoxolaner $\left(\operatorname{NexGard}^{\circledR}\right)$, administré par voie orale le jour 0 conformément aux instructions de l'étiquette. Les jours 1 , 14 et 28, chaque chien a été exposé à 60 femelles à jeun et 10 mâles de Phlebotomus perniciosus pendant une heure. À la fin de chaque période d'exposition, les phlébotomes ont été évalués en termes de viabilité et de statut alimentaire. Il n'y avait pas de différence statistique dans la mortalité $(0,0$ à 5,4 \%), ni dans le taux d'engorgement $(61,6$ à $78 \%)$ entre le groupe témoin et le groupe traité lors de toutes les évaluations après une heure. Après la collecte, les phlébotomes vivants gorgés et non gorgés ont été conservés aux fins d'évaluation de la viabilité 48 et 72 heures après l'exposition. Dans le groupe témoin non traité, le pourcentage moyen de phlébotomes femelles gorgées et vivantes après l'exposition aux jours 1,14 et 28 variait de 51 à $74 \%$ à 48 heures et de 46 à $57 \%$ à 72 heures, démontrant la robustesse du modèle au cours des 28 jours de l'étude. Un nombre significativement moins important de phlébotomes gorgés vivants ont été enregistrés dans le groupe traité par afoxolaner $(p<0,01)$. L'efficacité insecticide était de $100 \%$, $95,9 \%$ et $75,2 \% 48$ heures après les expositions des jours 1,14 et 28 , respectivement, et $100 \%, 100 \%$ et $86,3 \%$ à 72 heures après les expositions des jours 1, 14 et 28, respectivement. Une seule administration d'afoxolaner $\left(\operatorname{NexGard}^{\circledR}\right)$ par voie orale à un chien tue de manière significative les phlébotomes $P$. perniciosus 48 heures et 72 heures après la prise de sang pendant un mois.

\section{Introduction}

Canine leishmaniosis (CL) is an infectious disease due to the proliferation of the protozoan flagellate parasite Leishmania infantum in cells of the reticulo-endothelial

*Corresponding author: frederic. beugnet@merial.com system (i.e., monocyte cell line) [7]. This parasite is mainly transmitted by the bite of phlebotomine sandflies (Phlebotomus in the Old World, i.e., Africa, Asia, Europe; and Lutzomyia in the Americas) $[11,15,16,26]$. Leishmania protozoans can also be transmitted, rarely, by blood transfusion, or vertically from mothers to their puppies [28]. 
Although dogs constitute the main reservoir, L. infantum can also infect many other mammals like lagomorphs, rodents, foxes, cats, horses, and humans [15, 16, 26, 28]. CL is a major zoonosis and human cases are reported in endemic areas where the prevalence of CL in dogs is high $[16,28]$. Two hundred cases in Italy and approximately 25 autochthonous cases in France are reported yearly [27, 30].

Canine leishmaniosis is endemic in more than 70 countries. It is highly endemic in countries around the Mediterranean basin, but also West Africa, Southern Asia, and Central and South America. In endemic countries, the seroprevalence in dogs can vary greatly from a few percentage points to more than $50 \%$. Distribution can be highly heterogeneous between endemic foci (i.e., high seroprevalence in dogs, multiple clinical cases) and ectopic or new foci (i.e., low prevalence, few clinical cases and no or very few vectors) [15, 16, 26, 27].

Recent surveys have demonstrated a gradual spread to previously non-infected areas [10, 26, 27, 30]. Several authors have described new outbreaks from Southern or Central Italy to Northern Italy, such as Tuscany, Marche and EmiliaRomagna; in France with a spread to the West and Northwest [10]; as well as Catalonia, in northeastern Spain [1, 23], and Galicia, in northern Spain [25]. Canine leishmaniosis is becoming endemic in the Balkans and Romania, with extension towards Central and Northern Europe [23].

Phlebotomus perniciosus is one of the major vectors of canine leishmaniosis in Southern Europe [19, 20]. Other Phlebotomus species are also involved in North Africa, Southeastern Europe and Central Asia, e.g. Phlebotomus ariasi, $P$. perfiliewi, $P$. sergenti. $P$. perniciosus is a ubiquitous sandfly living in urban and peri-urban areas and having crepuscular activity [20]. P. perniciosus is a proven vector of this protozoan in Algeria, France, Italy, Malta, Portugal, Spain, Greece, and Turkey, and is a suspected vector in Morocco and Tunisia [24]. Over the past decades, there has been an increase in sandfly geographical distribution and density, which can be attributed to climate and ecological changes, but also to increased tourism [23, 24, 26].

There are two essential strategies to limit the transmission of L. infantum to dogs and humans: (1) control of the canine reservoir by using insecticides with repellent activity to prevent sandfly bites, by treating infected dogs, and by vaccinating dogs in enzootic areas; (2) control and reduce the vector density by acting on sandflies and/or sandfly ecosystems [26, 30].

Female sandflies take their blood meal in a short time, approximately $4 \mathrm{~min}$ and are able to inoculate Leishmania during that time $[2,9]$. Therefore, systemic insecticides will not prevent the infection of dogs during the sandfly bite. However, they may decrease the population of sandflies and avoid further bites and transmission to mammals because sandflies take 6-10 days to lay eggs before biting a new host again [13]. They also do not fly long distances (maximum $1 \mathrm{~km}$ ) and usually stay concentrated around 200-500 m [19, 20].

Afoxolaner is a systemic insecticide and acaricide compound from the isoxazoline group. Afoxolaner acts by inhibition of a specific receptor on GABA-gated chloride ion channels, resulting in uncontrolled activity of the central nervous system and death of the arthropods [28]. After oral administration, afoxolaner is rapidly absorbed and is highly bound to plasma proteins, therefore acting through a systemic pathway on hematophagous arthropods [21]. Afoxolaner is available as a palatable chew [18] given orally at the minimum dose of $2.5 \mathrm{mg} / \mathrm{kg}\left(\mathrm{NexGard}{ }^{\circledR}\right.$, Boehringer Ingelheim Animal Health). It is indicated for the treatment and control of fleas and ticks in dogs $[3,4,12,17]$, the treatment of Demodex and Sarcoptes [5, 6], and has been proven effective against Otodectes mites [8]. More recently, the insecticidal activity of afoxolaner against Aedes aegypti mosquitoes at $24 \mathrm{~h}$ postexposure was demonstrated. It showed that $A$. aegypti mosquitoes ingested a lethal dose of afoxolaner during their blood meal [22]. Based on these results, we hypothesized that afoxolaner could also kill sandflies that feed on afoxolaner treated dogs [13].

Afoxolaner has no repellent properties. Nevertheless, we hypothesized that it could be a good candidate in the control of sandfly populations by killing those that would feed on treated dogs. Killing female sandflies before a new bite and preventing them from laying eggs would help to reduce the sandfly population in a restricted area.

\section{Materials and methods}

The design and conditions of this study were approved by animal welfare ethical committees of Boehringer-Ingelheim and ClinVet, and were performed in accordance with International Cooperation on Harmonization of Technical Requirements for Registration of Veterinary Medicinal Products (VICH) Guideline 9, entitled Good Clinical Practice. The containment of the dogs also complied with the Directive 2010/63/EU of the European Parliament and of the Council of 22 September 2010 on the protection of animals used for scientific purposes, and was approved by the Institutional Animal Care and Use Committee (IACUC). This study was a parallel group, blinded, randomized, negative controlled efficacy study. It was conducted with two groups of six dogs each.

A total of 12 healthy laboratory Beagle dogs (six males and six females) between 14 and 18 months-old and weighing between 7.9 and $13.6 \mathrm{~kg}$ were included in the study. Dogs were acclimatized to the study conditions for seven days and examined by a veterinarian. The 12 dogs were randomly allocated to two groups of six dogs (untreated control group and afoxolaner treated group) based on body weight. All dogs were observed daily from acclimation start to the end of the study for general health. None of the dogs participating in this study had been treated with any acaricide/insecticide compound within three months preceding Day 0. The animals were kept individually in cages with visual and auditory contact with conspecifics. Dogs from the same group were allowed to access an outdoor shared exercise area from Day 7 onwards. At least one toy was made available to each dog (replenished weekly). The dog cages were part of a semi-indoor animal unit with a natural photoperiod.

On Day 0, NexGard ${ }^{\circledR}(2.27 \%$ w/w afoxolaner chewable tablets) was administered orally to all dogs assigned to the 
Table 1. Arithmetic means ( $\pm 95 \%$ confidence interval $[\mathrm{CI}], \alpha=0.05$ ) for female sandflies status at 1,48 , and $72 \mathrm{~h}$ post-exposure.

\begin{tabular}{|c|c|c|c|c|c|c|c|c|c|c|c|c|c|}
\hline \multirow[t]{3}{*}{ Group } & \multirow{3}{*}{$\begin{array}{c}\text { Hours } \\
\text { post- } \\
\text { exposure }\end{array}$} & \multicolumn{12}{|c|}{ Days of exposure and status of collected female sandflies } \\
\hline & & \multicolumn{4}{|c|}{ Day 1} & \multicolumn{4}{|c|}{ Day 14} & \multicolumn{4}{|c|}{ Day 28} \\
\hline & & Fed live & Fed dead & Unfed live & Unfed dead & Fed live & Fed dead & Unfed live & Unfed dead & Fed live & Fed dead & Unfed live & Unfed dead \\
\hline \multirow[t]{3}{*}{ Control } & $1 \mathrm{~h}$ & $37.8( \pm 8.5)$ & 0 (na) & $13.8( \pm 6.9)$ & $0.2( \pm 0.3)$ & $26.7( \pm 7.3)$ & $1.0( \pm 0.9)$ & $6.7( \pm 3.3)$ & $1.5( \pm 1.9)$ & $35.8( \pm 13.6)$ & $0.5( \pm 0.7)$ & $15.0( \pm 9.0)$ & $1.5( \pm 1.4)$ \\
\hline & $48 \mathrm{~h}$ & $24.5( \pm 5.2)$ & $13.3( \pm 7.0)$ & $12.0( \pm 6.1)$ & $2.0( \pm 1.2)$ & $15.0( \pm 7.3)$ & $12.7( \pm 2.3)$ & $4.7( \pm 3.1)$ & $3.5( \pm 2.5)$ & $26.3( \pm 9.4)$ & $10.0( \pm 5.4)$ & $11.3( \pm 7.7)$ & $5.2( \pm 4.7)$ \\
\hline & $72 \mathrm{~h}$ & $20.7( \pm 5.1)$ & $17.2( \pm 7.9)$ & $10.3( \pm 5.3)$ & $3.7( \pm 1.9)$ & $14.0( \pm 6.6)$ & $13.7( \pm 2.2)$ & $3.8( \pm 2.5)$ & $4.3( \pm 2.2)$ & $20.5( \pm 6.6)$ & $15.8( \pm 9.0)$ & $9.5( \pm 5.8)$ & $7.0( \pm 5.4)$ \\
\hline \multirow[t]{3}{*}{ Afoxolaner } & $1 \mathrm{~h}$ & $38.2( \pm 5.0)$ & 0 (na) & $9.5( \pm 4.9)$ & $1.8( \pm 2.3)$ & $26.5( \pm 7.0)$ & $1.2( \pm 1.2)$ & $8.8( \pm 5.8)$ & $3.3( \pm 1.6)$ & $34.7( \pm 12.0)$ & $0.7( \pm 0.4)$ & $16.3( \pm 10.4)$ & $4.8( \pm 2.9)$ \\
\hline & $48 \mathrm{~h}$ & 0 (na) & $38.2( \pm 5.0)$ & $5.0( \pm 3.5)$ & $5.3( \pm 3.9)$ & $0.7( \pm 0.7)$ & $27.0( \pm 5.9)$ & $6.0( \pm 5.6)$ & $6.2( \pm 2.7)$ & $7.7( \pm 10.6)$ & $27.7( \pm 11.8)$ & $10.7( \pm 7.8)$ & $10.5( \pm 3.6)$ \\
\hline & $72 \mathrm{~h}$ & 0 (na) & $38.2( \pm 5.0)$ & $4.0( \pm 2.4)$ & $6.3( \pm 4.7)$ & 0 (na) & $27.7( \pm 6.3)$ & $5.3( \pm 4.4)$ & $6.8( \pm 3.0)$ & $4.0( \pm 7.1)$ & $31.3( \pm 10.6)$ & $8.0( \pm 5.0)$ & $13.2( \pm 5.4)$ \\
\hline
\end{tabular}

na, not applicable.

treated group in accordance with European label instructions. On Day 1, 14 and 28, dogs were exposed to 60 unfed females and 10 males $P$. perniciosus, in a dark room, in a dark room for $60 \mathrm{~min}$ ( $\pm 5 \mathrm{~min})$. The sandfly numbers varied for each dog and each challenge, but the exact counts were performed at collection time $1 \mathrm{~h}$ after each exposure. For the sandfly exposure, each dog was sedated using medetomidine (Domitor ${ }^{\circledR}$, Zoetis) and the head of the dog was placed into a sandfly proof net (dimensions: $40 \mathrm{~cm} \times 40 \mathrm{~cm} \times 40 \mathrm{~cm}$ ). Although males do not take blood meals, their presence improves female engorgement [28]. At the end of each 1-h exposure period, sandflies were smoothly vacuumed from the enclosure, categorized (male/female), counted and assessed for viability status (live/dead). As classically performed for insects and acarians, moribund sandflies were counted as live, which is more restrictive in the assessment of effectiveness [22]. Sandflies were also categorized as either fed or unfed, separated, and transferred to containers. All live fed female sandflies were then kept and incubated in vials at approximately $25{ }^{\circ} \mathrm{C}$ and $>60 \%$ relative humidity to perform further viability assessments (live, dead) at 48 and $72 \mathrm{~h}$ after each exposure.

A laboratory-bred strain of $P$. perniciosus originating from Italy was used for the exposures [29]. Sandflies were unfed and aged from 3 to 10 days on the day of challenge.

The efficacy of afoxolaner against $P$. perniciosus was calculated using the total number of live fed female sandflies at 1,48 and $72 \mathrm{~h}$ after each exposure, according to the formula below:

Insecticidal efficacy (\%) against sandflies

$$
=100 \times\left(P_{c}-P_{t}\right) / P_{c},
$$

where $P_{c}=$ Arithmetic mean number of the proportion* of live fed female sandflies in the control group; $P_{t}=$ Arithmetic mean number of the proportion* of live fed female sandflies in the treated group;

$$
\begin{aligned}
& * \text { Proportion of live fed sandflies per animal } \\
& \quad=[(\text { Live fed sandflies } /(\text { Live }+ \text { Dead }) \text { fed sandflies })] .
\end{aligned}
$$

In addition, feeding proportion (at each 1-h post-exposure) and mortality $\%$ (1, 48 and $72 \mathrm{~h}$ post-exposure) were calculated for each control dog, according to the formulas below:
Feeding proportion $[\%]=$

[(Total fed sandflies/Total collected sandflies $) \times 100]$,

Mortality $[\%]=$

[(Total dead fed sandflies/Total collected fed sandflies $) \times 100]$.

The groups were compared using a Wilcoxon Sum Rank Test. SAS Version 9.3 TS Level 1M2 was used for the statistical analyses.

\section{Results}

No adverse event was recorded after treatment or during the study duration.

The live or dead status, as well as the engorgement status, of the sandflies was assessed at all time-points (Table 1). The mortality observed in the control group on Days 1, 14 and 28 after the 1-h exposure ranged from $0.0 \%$ to $3.3 \%$ (Table 2) and the feeding proportion ranged from $66 \%$ to $77 \%$ (Table 3 ), indicating that the sandfly strain was vigorous and that the feeding model worked. The mortality observed in the treated group on Days 1, 14 and 28 after the 1-h exposure ranged from $0.0 \%$ to $5.4 \%$ (Table 2) and the feeding proportion ranged from $61.6 \%$ to $78 \%$ (Table 3). There was no statistical difference in the mortality rate, nor in the feeding proportion between the control and the treated groups at all 1 -h post-exposure assessments for all time-points.

In the control group, sandfly mortality was $33.3 \%, 49.3 \%$ and $26.3 \%$ at $48 \mathrm{~h}$ and $43.5 \%, 52.5 \%$ and $39.5 \%$ at $72 \mathrm{~h}$ post-exposure on Days 1, 14 and 28, respectively. In the treated group, $100 \%$ of the fed sandflies were dead at the $48 \mathrm{~h}$ assessment on Day 1, $100 \%$ at $72 \mathrm{~h}$ on Day 14 and $91.7 \%$ at $72 \mathrm{~h}$ on Day 28 (Table 2).

Significantly fewer live fed sandflies were recorded for the afoxolaner treated group compared to the negative control group, both at 48 and $72 \mathrm{~h}$ assessments, after each exposure on Days 1,14 and 28 ( $p<0.01)$ (Table 4$)$.

The afoxolaner insecticidal efficacy against $P$. perniciosus was $100 \%, 95.9 \%$ and $75.2 \%$ at 48 h post day 1,14 and 28 challenges, and $100 \%, 100 \%$ and $86.3 \%$ at $72 \mathrm{~h}$ post day 1 , 14, and 28 challenges (Table 4). 
Table 2. Mortality $\%$ of fed female sandflies at 1,48 , and $72 \mathrm{~h}$ postexposure.

\begin{tabular}{|c|c|c|c|c|}
\hline \multirow[t]{3}{*}{ Group } & \multirow[t]{3}{*}{ Day } & \multicolumn{3}{|c|}{ Hours post-exposure } \\
\hline & & $1 \mathrm{~h}$ & $48 \mathrm{~h}$ & $72 \mathrm{~h}$ \\
\hline & & Mortality (\%) & Mortality (\%) & Mortality (\%) \\
\hline \multirow[t]{3}{*}{ Control } & D1 & 0.0 & 33.3 & 43.5 \\
\hline & D14 & 3.3 & 49.3 & 52.5 \\
\hline & D28 & 0.9 & 26.3 & 39.5 \\
\hline \multirow{3}{*}{ Afoxolaner } & D1 & 0.0 & 100.0 & 100.0 \\
\hline & D14 & 5.4 & 97.9 & 100.0 \\
\hline & D28 & 2.6 & 81.8 & 91.7 \\
\hline
\end{tabular}

Mortality $[\%]=[($ Total dead fed sandflies/Total collected fed sandflies) $\times 100]$.

Table 3. Feeding proportion of female sandflies at $1 \mathrm{~h}$ postexposure.

\begin{tabular}{lcc}
\hline Group & Day & Feeding proportion (\%) \\
\hline Control & D1 & 72.8 \\
& D14 & 77.0 \\
Afoxolaner & D28 & 66.0 \\
& D1 & 78.0 \\
& D14 & 69.6 \\
& D28 & 61.6 \\
\hline
\end{tabular}

Feeding proportion $[\%]=[($ Total fed sandflies/Total collected sandflies) $\times 100]$.

Table 4. Average proportions of live fed sandflies and insecticidal efficacy at 1, 48 and $72 \mathrm{~h}$ post-exposure.

\begin{tabular}{|c|c|c|c|c|c|}
\hline \multirow[t]{2}{*}{ Day } & \multirow[t]{2}{*}{$\begin{array}{c}\text { Hours } \\
\text { post-exposure }\end{array}$} & \multicolumn{2}{|c|}{$\begin{array}{l}\text { Average proportion } \\
\text { of live fed sandflies } \\
\quad \pm 95 \% \mathrm{CI}\end{array}$} & \multirow[t]{2}{*}{$\begin{array}{l}\text { Insecticidal } \\
\text { efficacy }(\%)\end{array}$} & \multirow[t]{2}{*}{$p$-value** } \\
\hline & & $\begin{array}{l}\text { Control } \\
\text { group }\end{array}$ & $\begin{array}{l}\text { Afoxolaner } \\
\text { group }\end{array}$ & & \\
\hline \multirow[t]{3}{*}{1} & $1 \mathrm{~h}$ & $100^{*}$ & $100 *$ & - & \\
\hline & $48 \mathrm{~h}$ & $66.7 \pm 13.6$ & $0 *$ & $100 \%$ & $p<0.0027$ \\
\hline & $72 \mathrm{~h}$ & $56.5 \pm 13.1$ & $0 *$ & $100 \%$ & $p<0.0027$ \\
\hline \multirow[t]{3}{*}{14} & $1 \mathrm{~h}$ & $96.7 \pm 2.5$ & $94.6 \pm 6.0$ & - & \\
\hline & $48 \mathrm{~h}$ & $50.7 \pm 213.6$ & $2.1 \pm 1.9$ & $95.9 \%$ & $p<0.0047$ \\
\hline & $72 \mathrm{~h}$ & $47.5 \pm 311.8$ & $0 *$ & $100 \%$ & $p<0.0047$ \\
\hline \multirow[t]{3}{*}{28} & $1 \mathrm{~h}$ & $99.1 \pm 1.2$ & $97.4 \pm 2.1$ & - & \\
\hline & $48 \mathrm{~h}$ & $73.7 \pm 6.6$ & $18.2 \pm 21.2$ & $75.2 \%$ & $p<0.0129$ \\
\hline & $72 \mathrm{~h}$ & $60.5 \pm 12.2$ & $8.3 \pm 14.4$ & $86.3 \%$ & $p<0.0043$ \\
\hline
\end{tabular}

Proportion of live fed sandflies per animal $[\%]=[($ Live fed sandflies/Total fed sandflies $) \times 100]$.

Insecticidal efficacy $(\%)$ against sandflies $=100 \times\left(P_{c}-P_{t}\right) / P_{c}$, where $P_{c}=$ Arithmetic mean number of the proportion of live fed female sandflies in the control group; $P_{t}=$ Arithmetic mean number of the proportion of live fed female sandflies in the treated group.

${ }^{*}$ For all samples showing 0 or $100 \%$ live sandflies, there is no $95 \%$ CI.

** $p$-value based on a Wilcoxon sum rank test on live fed female counts between groups at each time-point.

\section{Discussion and conclusions}

The study demonstrated the insecticidal activity, thus the mortality of female sandflies, after a blood meal on afoxolaner treated dogs. Female sandflies take their blood meal in a short time, approximately $4 \mathrm{~min}$ [9], and they ingest $4-5 \mu \mathrm{L}$ of blood [2, 11]. Proteins provided by the blood meal are necessary for egg production $[19,20]$. P. perniciosus eggs are laid 6-10 days after a blood meal, and before the next blood meal [29]. With a limited volume of blood and therefore a low quantity of afoxolaner ingested, we did not expect an immediate killing effect, but did expect the death within several hours or days after feeding, so before the females lay eggs or bite a second time. Leishmania promastigotes need 7 to 10 days to become infective in the female sandfly [11, 19]. Therefore treating dogs having canine leishmaniosis would prevent transmission to other dogs. Phlebotomus sandflies do not fly long distances (maximum $1 \mathrm{~km}$ ) and stay usually concentrated around $200-500 \mathrm{~m}$. Therefore, the biological features are in favor of the possibility of obtaining a decrease in the sandfly population biting afoxolaner treated dogs, thereby possibly reducing the rate of Leishmania transmission in endemic areas [13].

In this study, the observed mortality of sandflies in the control group at $1 \mathrm{~h}$ post exposure was low (less than $3.3 \%$ after each challenge) and the feeding proportion was higher than $66 \%$, demonstrating the relevance of the experimental model.

A single administration of afoxolaner was enough to kill $100 \%$ of sandflies within $48 \mathrm{~h}$ of exposure on Day 1, 96\% and $100 \%$ of sandflies within $48 \mathrm{~h}$ and $72 \mathrm{~h}$ of exposure on Day 14; and 75.0 and $86.4 \%$ of sandflies within $48 \mathrm{~h}$ and $72 \mathrm{~h}$ of exposure on Day 28, respectively. Recently another isoxazoline, fluralaner, also demonstrated efficacy against Phlebotomus papatasi sandflies after oral administration to dogs [14]. The authors reached a similar conclusion on the beneficial activity of systemic isoxazolines for the control of vector populations.

Isoxazolines, like afoxolaner or fluralaner, do not provide prevention of Leishmania transmission as the female sandflies bite before dying. Therefore, preventative measures like repellent application or vaccination are still needed for individual dog protection. Nevertheless, the insecticidal activity of systemically active isoxazolines against the vector is an additional beneficial measure to decrease vector density and decrease risk at the population level.

\section{Competing interest}

The work reported herein was funded by Boehringer-Ingelheim Animal Health. Wilfried Lebon and Frédéric Beugnet are current employees of Boehringer-Ingelheim. Nadège Perier and Nesrine Aouiche are veterinarians, finishing a Master 2 degree in Science at Lyon University. Leon Meyer and Noua Lekouch are employees of the CRO ClinVet.

NEXGARD $^{\circledR}$ is a registered trademark of Merial, now part of Boehringer-Ingelheim. All other marks are the property of their respective owners.

This document is provided for scientific purposes only. Any reference to a brand or trademark herein is for informational purposes only and is not intended for a commercial purpose or to dilute the rights of the respective owner(s) of the brand(s) and trademark(s). 


\section{References}

1. Ballart C, Alcover MM, Picado A, Nieto J, Castillejo S, Portus M, Gallego M. 2013. First survey on canine leishmaniasis in a non classical area of the disease in Spain (Lleida, Catalonia) based on a veterinary questionnaire and a cross-sectional study. Preventive Veterinary Medicine, 109, 116-127.

2. Bergman DK. 1996. Mouthparts and feeding mechanisms of haematophagous arthropods, in The immunology of hostectoparasitic arthropod relationships, Wikel SK, Editor. CAB International: Wallingford, UK. p. 38-45.

3. Beugnet F, Liebenberg J, Halos L. 2015. Comparative efficacy of two oral treatments for dogs containing either afoxolaner or fluralaner against Rhipicephalus sanguineus sensu lato and Dermacentor reticulatus. Veterinary Parasitology, 209, 142-145.

4. Beugnet F, Liebenberg J, Halos L. 2015. Comparative speed of efficacy against Ctenocephalides felis of two oral treatments for dogs containing either afoxolaner or fluralaner. Veterinary Parasitology, 207, 297-301.

5. Beugnet F, de Vos C, Liebenberg J, Halos L, Larsen D, Fourie J. 2016. Efficacy of afoxolaner in a clinical field study in dogs naturally infested with Sarcoptes scabiei. Parasite, 23, 26.

6. Beugnet F, Halos L, Larsen D, de Vos C. 2016. Efficacy of oral afoxolaner for the treatment of canine generalised demodicosis. Parasite, 23, 14.

7. Beugnet F, Halos L, Guillot J. 2018. Textbook of clinical parasitology in dogs and cats. Zaragoza, Spain: Servet Editions. p. $166-174$.

8. Carithers D, Crawford J, de Vos C, Lotriet A, Fourie J. 2016. Assessment of afoxolaner efficacy against Otodectes cynotis infestations of dogs. Parasites \& Vectors, 9, 635.

9. Colange H. 2011. Contribution à l'étude du repas sanguin de Phlebotomus perniciosus (Diptera : Psychodidae). Toulouse, France: Thèse d'exercice, Médecine vétérinaire, École Nationale Vétérinaire de Toulouse - ENVT, 78 p.

10. Dereure J, Vanwambeke SO, Malé P, Martinez S, Pratlong F, Balard Y, Dedet JP. 2009. The potential effects of global warming on changes in canine leishmaniasis in a focus outside the classical area of the disease in southern France. VectorBorne Zoonotic Diseases, 9, 687-694.

11. Dolmatova AV, Demina NA. 1971. Les phlébotomes (Phlebotominae) et les maladies qu'ils transmettent. Paris, France: Office de la Recherche Scientifique et Technique d'Outre Mer (ORSTOM), $168 \mathrm{p}$.

12. European Medicines Agency. 2014. Nexgard ${ }^{\circledR}$ summary of product characteristics. http://www.ema.europa.eu/docs/en_GB/ document_library/EPAR_-_Product_Information/veterinary/ 002729/WC500164067.pdf.

13. Gomez SA, Picado A. 2017. Systemic insecticides used in dogs: potential candidates for phlebotomine vector control? Tropical Medicine \& International Health, 22, 755-764.

14. Gomez SA, Lucientes J, Castillo JA, Peris MP, Delacour S, Ortega P, Oropeza RV, Picado A. 2018. A randomized, blinded, controlled trial to assess sand fly mortality of fluralaner administered orally in dogs. Parasites \& Vectors, 11, 627.
15. Grammicia M. 2011. Recent advances in leishmaniosis in pet animals: epidemiology, diagnostics and anti-vectorial prophylaxis. Veterinary Parasitology, 8, 23-30.

16. Grammicia M, Gradoni L. 2005. The current status of zoonotic leishmaniases and approaches to disease control. International Journal for Parasitology, 35, 1169-1180.

17. Halos L, Lebon W, Chalvet-Monfray K, Larsen D, Beugnet F. 2014. Immediate efficacy and persistent speed of kill of a novel oral formulation of afoxolaner $\left(\mathrm{NexGard}^{\circledR}\right)$ against induced infestations with Ixodes ricinus ticks. Parasites \& Vectors, 7, 452.

18. Halos L, Carithers DS, Solanki R, Stanford H, Gross SJ. 2015. Preference of dogs between two commercially available oral formulations of ectoparasiticide containing isoxazolines, afoxolaner or fluralaner. Scientific Research Publishing, 5, 25-29.

19. Killick-Kendrick R. 1979. Biology of Leishmania in phlebotomine sandflies, in Biology of the Kinetoplastida, Lumsden WH, Evans DA, Editors. Academic Press: London. p. 395-460.

20. Killick-Kendrick R. 1999. The biology and control of Phlebotomine sand flies. Clinics in Dermatology, 17, 279-289.

21. Letendre L, Huang R, Kvaternick V, Harriman J, Drag M, Soll M. 2014. The intravenous and oral pharmacokinetics of afoxolaner used as a monthly chewable antiparasitic for dogs. Veterinary Parasitology, 201, 190-197.

22. Liebenberg J, Fourie J, Lebon W, Larsen D, Halos L, Beugnet F. 2017. Assessment of the insecticidal activity of afoxolaner against Aedes aegypti in dogs treated with NexGard $^{\circledR}$. Parasite, 24, 39.

23. Maia C, Cardoso L. 2015. Spread of Leishmania infantum in Europe with dog travelling. Veterinary Parasitology, 30, 2-11.

24. Maroli M, Feliciangeli MD, Bichaud L, Charrel RN, Gradoni L. 2013. Phlebotomine sandflies and the spreading of leishmaniases and other diseases of public health concern. Medical and Veterinary Entomology, 27, 123-147.

25. Miró G, Checa R, Montoya A, Hernandez L, Dado D, Galvez R. 2012. Current situation of Leishmania infantum infection in shelter dogs in northern Spain. Parasites \& Vectors, 5, 60.

26. Otranto D, Dantas-Torres F. 2013. The prevention of canine leishmaniasis and its impact on public health. Trends in Parasitology, 29, 339-345.

27. Palatnik-de-Soussa CB, Day MJ. 2011. One health: the global challenge of epidemic and endemic leishmaniasis. Parasite \& Vectors, 4, 197.

28. Shoop WL, Hartline EJ, Gould BR, Waddell ME, McDowell RG, Kinney JB, Lahm GP, Long JK, Xu M, Wagerle T, Jones GS, Dietrich RF, Cordova D, Schroeder ME, Rhoades DF, Benner EA, Confalone PN. 2014. Discovery and mode of action of afoxolaner, a new isoxazoline parasiticide for dogs. Veterinary Parasitology, 201, 179-189.

29. Volf P, Volfova V. 2011. Establishment and maintenance of sand fly colonies. Journal of Vector Ecology, 36, S1-S9.

30. World Health Organization. 2010. Control of the leishmaniasis: report of a meeting of the WHO Expert Committee on the Control of Leishmaniases. Geneva. (WHO Technical Report Series, 949).

Cite this article as: Perier N, Lebon W, Meyer L, Lekouch N, Aouiche N \& Beugnet F. 2019. Assessment of the insecticidal activity of oral afoxolaner against Phlebotomus perniciosus in dogs. Parasite 26, 63. 
Reviews, articles and short notes may be submitted. Fields include, but are not limited to: general, medical and veterinary parasitology; morphology, including ultrastructure; parasite systematics, including entomology, acarology, helminthology and protistology, and molecular analyses; molecular biology and biochemistry; immunology of parasitic diseases; host-parasite relationships; ecology and life history of parasites; epidemiology; therapeutics; new diagnostic tools.

All papers in Parasite are published in English. Manuscripts should have a broad interest and must not have been published or submitted elsewhere. No limit is imposed on the length of manuscripts.

Parasite (open-access) continues Parasite (print and online editions, 1994-2012) and Annales de Parasitologie Humaine et Comparée (1923-1993) and is the official journal of the Société Française de Parasitologie. 